\title{
El respeto a las normas de tránsito en la cuidad de Santa Fe, Argentina
}

\author{
Juan Carlos Beltramino ${ }^{1}$ y Elena Carrera ${ }^{2}$
}

Forma de citar

Beltramino JC, Carrera E. El respeto a las normas de tránsito en la cuidad de Santa Fe, Argentina. Rev Panam Salud Publica. 2007;22(2):141-5.

RESUMEN Se realizó un estudio de campo observacional descriptivo para evaluar el cumplimiento de algunas normas de tránsito en la ciudad de Santa Fe, Argentina, y comparar los resultados con mediciones realizadas anteriormente. Para ello, entre el 2 y el 4 de enero de 2006, se verificó en 13 sitios de la ciudad el cumplimiento de las normas que obligan a los automovilistas a usar cinturón de seguridad y a llevar a los menores de 10 años en el asiento trasero, y a los motociclistas, a conducir con casco y a no transportar menores. Las observaciones abarcaron 4173 automóviles y 1013 motocicletas. Solo 9\% de los conductores de automóviles usaban el cinturón de seguridad. En 56\% de los 246 automóviles que transportaban menores, estos se hallaban en el asiento delantero. En cuanto a los motociclistas, solo $12 \%$ usaba casco y 6,7\% transportaba niños. Al comparar estas observaciones con cinco registros anuales anteriores, se comprobó que en los últimos tres años disminuyeron los porcentajes de acatamiento de estas normas de tránsito. Los resultados de este estudio sugieren que la mayoría de los conductores de vehículos de la ciudad argentina de Santa Fe viola las normas de tránsito consideradas. Para lograr disminuir la elevada mortalidad por accidentes viales no basta con contar con leyes de tránsito y organizar campañas de información, sino que, además, es necesario implementar sistemas que vigilen el cumplimiento de las normas.

Palabras clave Accidentes de tránsito, prevención de accidentes, cinturones de seguridad, Argentina.

La asociación civil argentina Luchemos por la Vida informó que durante el año 2005 murieron en el país por accidentes de tránsito 7138 personas (1), lo que equivale a 595 muertes por mes o 20 cada 24 horas. La provincia de

\footnotetext{
1 Hospital de Niños Dr. Orlando Alassia, Santa Fe, Provincia de Santa Fe, Argentina. Toda correspondencia deberá dirigirse a: Juan Carlos Beltramino, Hospital de Niños Dr. Orlando Alassia, Dirección asociada de docencia e investigación, Mendoza 4151, (3000) Santa Fe, Provincia de Santa Fe, Argentina; tel.: (342) 4505992; correo electrónico: jcbeltramino@yahoo.com.ar.

2 Universidad Nacional del Litoral, Facultad de Bioquímica y Ciencias Biológicas, Laboratorio de Bioestadística, Santa Fe, Provincia de Santa Fe, Argentina.
}

Santa Fe, donde se notificaron 552 de esas muertes, ocupa el segundo lugar del país en cantidad de casos, detrás de la provincia de Buenos Aires.

Los accidentes de tránsito en general son la primera causa de muerte entre los niños mayores de un año y los adolescentes argentinos (2). En el año 2005 ingresaron al Servicio de Urgencias del Hospital de Niños Dr. Orlando Alassia (HNOA) de la ciudad de Santa Fe 367 pacientes con lesiones por accidentes de tránsito, es decir, un niño cada 24 horas (Informe del Departamento de Epidemiología del HNOA, enero de 2006). Este número triplicó el de los ingresos por la misma causa del año 2000. En el país, en 2004, casi la mitad de los muertos por accidentes viales fueron menores de 30 años (3).

Si una enfermedad infecciosa hubiese causado $1 \%$ de esas muertes, los funcionarios, los periodistas y la sociedad toda reclamarían a las autoridades sanitarias conocer cuáles son las causas y las medidas que deben tomarse para limitar el desastre, y se consultarían expertos para aplicar las acciones preventivas más eficaces. Sin embargo, con respecto a la "enfermedad 
accidentes de tránsito", en Argentina, pareciera que las autoridades sanitarias sólo deben ocuparse de las consecuencias de los accidentes, es decir, de tratar a los heridos y rehabilitarlos, ya que, aparentemente, ninguna medida preventiva es válida.

Desde 1995, la Municipalidad de Santa Fe obliga a asistir a un curso teórico y práctico para obtener el permiso de conductor. Desde entonces, miles de santafesinos han aprendido que para circular en un automóvil hay que colocarse el cinturón de seguridad, que los menores no deben llevarse en los asientos delanteros, que los motociclistas deben usar casco y que es obligatorio respetar las limitaciones de velocidad y los semáforos, entre otras normas tan importantes como las mencionadas. Todas estas disposiciones están contempladas en la Ley N. ${ }^{\circ}$ 24.449, llamada Ley de Tránsito. La provincia de Santa Fe se adhirió a esta ley en el año 1998 y, por lo tanto, debería hacerla cumplir.

En 1998, se levantaron en la ciudad de Santa Fe 123050 actas por infracciones de tránsito, de las cuales, 94604 fueron por mal estacionamiento, 8817 por problemas de documentación, 11153 por cruzar un semáforo en rojo, 2513 por conducir a velocidad peligrosa y 2739 por conducir a contramano (C. Collins, Director del Tribunal Municipal de Faltas de la ciudad de Santa Fe, comunicación personal, 1999).

Según estas cifras, de cada 10 sanciones, más de ocho tuvieron como objetivo castigar al "automóvil parado" y menos de dos, sancionar al "automóvil en movimiento". Sin embargo, la mayoría de las muertes y lesiones se deben a vehículos en movimiento.

Además de promulgar las leyes adecuadas y divulgarlas, es necesario verificar que éstas sean acatadas por la comunidad. Si se respetase la Ley de Tránsito, es probable que el número de accidentes no fuese tan elevado como en la actualidad. Surge así la hipótesis de que la comunidad santafesina no cumple ajustadamente estas normas de tránsito. Para verificarla se realizó un estudio de campo observacional descriptivo. Los objetivos de este estudio fueron:
1. Evaluar el cumplimiento de algunas normas de tránsito por parte de los conductores de vehículos en la ciudad de Santa Fe, Argentina.

2. Comparar los resultados obtenidos con mediciones realizadas en años anteriores.

\section{MATERIALES Y MÉTODOS}

Entre el 2 y el 4 de enero de 2006, se realizó en la ciudad de Santa Fe un registro visual del acatamiento de las normas de tránsito vigentes por parte de conductores de automóviles y motocicletas. Para ello, se llevaron a cabo observaciones con luz natural, en días soleados, en doce sitios predeterminados de la ciudad, a fin de registrar el cumplimiento, por parte de los automovilistas, de las normas que obligan a usar el cinturón de seguridad y a transportar a los menores de 10 años en el asiento trasero, y por parte de los motociclistas, de la obligación de conducir con casco y no transportar menores.

Se establecieron puestos de observación en cada uno de los doce sitios seleccionados, en lugares donde el tránsito es más lento debido a la cercanía de semáforos o a la presencia de reductores de velocidad o bifurcaciones. Se seleccionaron así la Plaza Colón, los hospitales Dr. Juan Bautista Iturraspe, Dr. Gumersindo Sayago, Dr. José María Cullen y Dr. Orlando Alassia, el Rectorado de la Universidad Nacional de Litoral (UNL), el Colegio Adoratrices, la Legislatura, la Facultad Regional Santa Fe de la Universidad Tecnológica Nacional (UTN), la Fuente de la Cordialidad, el Parque Juan de Garay y la Costanera, precisamente en la unión de las avenidas denominadas Costanera Vieja y Costanera Nueva.

Se decidió limitar el tiempo de observación a treinta minutos en cada sitio, excepto en el puesto de la Costanera, donde se extendió hasta una hora. En total, se realizaron observaciones durante siete horas, repartidas en tres días.

Los datos obtenidos se registraron en formularios y se procesaron con el programa informático SPSS, versión 10.0. La comparación de proporciones se realizó a través del estadístico $Z$, utilizando la distribución normal como aproximación de la binomial. En todos los casos se adoptó un nivel de significación $\alpha=0,05$.

\section{Criterios de inclusión y definición de términos}

Se explican a continuación los términos y las expresiones empleados en este estudio.

Automóviles: se incluyeron automóviles particulares, automóviles pertenecientes a empresas públicas y privadas, o a organismos públicos, camionetas, taxis y autos de alquiler con chofer para trayectos cortos (denominados en la Argentina "remises"), automóviles policiales, ambulancias y vehículos de transporte escolar.

Cinturón sí: cuando el conductor llevaba colocado el cinturón de seguridad, aunque no lo utilizaran los acompañantes.

Cinturón no: cuando el conductor no llevaba colocado el cinturón de seguridad, aunque lo utilizaran los acompañantes.

Menores en el automóvil: cuando en el automóvil se transportaban niños, desde lactantes hasta escolares de 10 años (según el criterio del observador); se anotó si los niños se hallaban en el asiento delantero o en el trasero.

Teléfonos móviles: se registró si el conductor del vehículo tenía un teléfono móvil en la mano.

Motocicletas: se incluyeron todas las motocicletas, desde los ciclomotores hasta las de mayor cilindrada.

Casco sí: cuando el motociclista llevaba el casco colocado en la cabeza, independientemente del comportamiento del eventual acompañante.

Menor en motocicleta: cuando la motocicleta transportaba uno o más niños, desde lactantes hasta escolares menores de 12 años (según el criterio del observador).

Carros: se incluyeron los carros tirados por caballos, utilizados por personas que viven de comercializar la basura que recogen. 
Observador: la persona que realizó las observaciones; en todos los casos, se trató de la misma persona, que ya había realizado anteriormente cinco registros (en 1999, 2000, 2001, 2002, 2004 y 2005) empleando una metodología similar.

\section{Criterios de exclusión}

No se incluyeron los camiones ni los ómnibus. No se registraron los casos donde se dudó si el conductor tenía colocado el cinturón de seguridad. Tampoco se incluyeron los vehículos con vidrios muy opacos, polarizados y cerrados, aunque se consignó su número.

\section{RESULTADOS}

En el cuadro 1 se resumen los resultados de los registros realizados. Como datos accesorios, entre los 3617 automovilistas observados (sin considerar a los taxistas), se detectaron 37 que hablaban por teléfono móvil mientras conducían. También se registró el paso por las calles de 14 carros. Por último, aunque no se contabilizaron, se observaron motocicletas que transportaban tres o más personas, incluidos niños.

\section{DISCUSIÓN}

Si bien la eficacia de los cinturones de seguridad para disminuir los traumatismos en caso de accidente automovilístico ha sido ampliamente comprobada (4), la Ley de Tránsito argentina prescribe su empleo y la Municipalidad de Santa Fe reitera la obligatoriedad de su uso en los cursos reglamentarios para conducir, este estudio demuestra que la mayoría de los conductores santafesinos no los utilizan. En efecto, sólo 9\% de los conductores observados llevaba el cinturón colocado, cifra muy inferior a la registrada en Argentina en el año 2003, donde la proporción de personas que utilizaban cinturones de seguridad en los asientos delanteros era alrededor

CUADRO 1. Registro sobre el cumplimiento de normas de tránsito por parte de los conductores en la ciudad de Santa Fe, Argentina, 2006

\begin{tabular}{|c|c|c|c|c|c|}
\hline & \multicolumn{2}{|c|}{ Sí } & \multicolumn{2}{|c|}{ No } & \multirow[b]{2}{*}{ Total } \\
\hline & $n$ & $(\%)$ & $n$ & $(\%)$ & \\
\hline \multicolumn{6}{|l|}{ Automóviles } \\
\hline Conductor visibles & 4173 & (93) & 300 & (7) & 4473 \\
\hline Cinturón de seguridad & 375 & (9) & 3798 & (91) & 4173 \\
\hline Vehículos particulares & 328 & (9) & 3209 & (91) & 3537 \\
\hline Taxis & 45 & (8) & 511 & (92) & 556 \\
\hline Vehículos policiales & 0 & (0) & 33 & $(100)$ & 33 \\
\hline Vehículos oficiales ${ }^{a}$ & 0 & (0) & 21 & $(100)$ & 21 \\
\hline Ambulancias & 1 & (5) & 17 & (95) & 18 \\
\hline Transportes escolares & 1 & (13) & 7 & (87) & 8 \\
\hline Menores en el asiento traserob & 108 & (44) & 138 & (56) & 246 \\
\hline \multicolumn{6}{|l|}{ Motocicletas } \\
\hline Casco & 122 & (12) & 891 & (88) & 1013 \\
\hline Menores (uno o más) & 68 & (7) & 925 & (93) & 1013 \\
\hline
\end{tabular}

a Vehículos identificados como pertenecientes a organismos oficiales, nacionales, provinciales y municipales, y a empresas. ${ }^{b}$ Niños observados en automóviles particulares.

de $26 \%$ en la ciudad de Buenos Aires y de $58 \%$ en las carreteras nacionales (5).

Según los resultados obtenidos, los conductores de vehículos oficiales y de taxis (reglamentados por la Municipalidad) circulan sin respetar esta norma de seguridad vial y tampoco la respetan quienes manejan automóviles de medios de comunicación, que diariamente difunden noticias sobre accidentes viales.

Es necesario emplear todas las medidas eficaces para incrementar el uso del cinturón. Todos los nuevos vehículos deberían tener una alarma sonora que sólo se desactivase si el conductor se lo ajusta. Estos dispositivos acústicos son una medida poco costosa de eficacia probada para aumentar el empleo del cinturón $(6,7)$.

En 56\% de los automóviles observados que transportaban menores había uno o más niños en el asiento delantero, a pesar de que la Ley de Tránsito establece que los menores de 10 años deben viajar siempre en el asiento trasero (8).

Está demostrado que cuando se transportan en un automóvil niños pequeños, más de $50 \%$ de las muertes infantiles por accidentes de tránsito podrían evitarse si éstos viajaran en el asiento trasero y en sillas de seguridad $(9,10)$. El riesgo aumenta significativamente cuando los niños son transpor- tados sin sujeción alguna y en el asiento delantero.

En 7\% de los vehículos observados en este estudio, el exceso de oscurecimiento de los vidrios impidió visualizar el interior. Además de dificultar la evaluación, los vidrios muy oscuros entorpecen la percepción visual de las personas que se hallan cerca del vehículo, las cuales podrían ajustar mejor sus movimientos si observasen el comportamiento del conductor.

Se estima que hablar por teléfono o recibir una llamada mientras se conduce cuadruplica la probabilidad de sufrir un accidente $(11,12)$. No obstante, $1 \%$ de los conductores santafesinos observados en este estudio hablaba por teléfono móvil. Esta cifra, aunque es menor que la registrada en la ciudad de Buenos Aires, de 2,1\% (13), aumentó en $100 \%$ con respecto a las observaciones realizadas en la ciudad de Santa Fe en 2005 (Informe de la Dirección de Docencia e Investigación del HNOA, "Poco respeto a las normas de tránsito en Santa $\mathrm{Fe}^{\prime \prime}$, 2005).

Entre los motociclistas, el porcentaje que circulaba con casco (12\%) fue muy inferior $(P<0,0001)$ al estimado en la ciudad de Buenos Aires, donde alcanzó $56 \%$ (14).

El uso del casco es la estrategia más exitosa para prevenir traumatismos en los conductores de motocicletas (15). 
FIGURA 1. Evolución del uso del casco (motociclistas) y del cinturón de seguridad (automovilistas) en la ciudad de Santa Fe, Argentina, 1999-2006

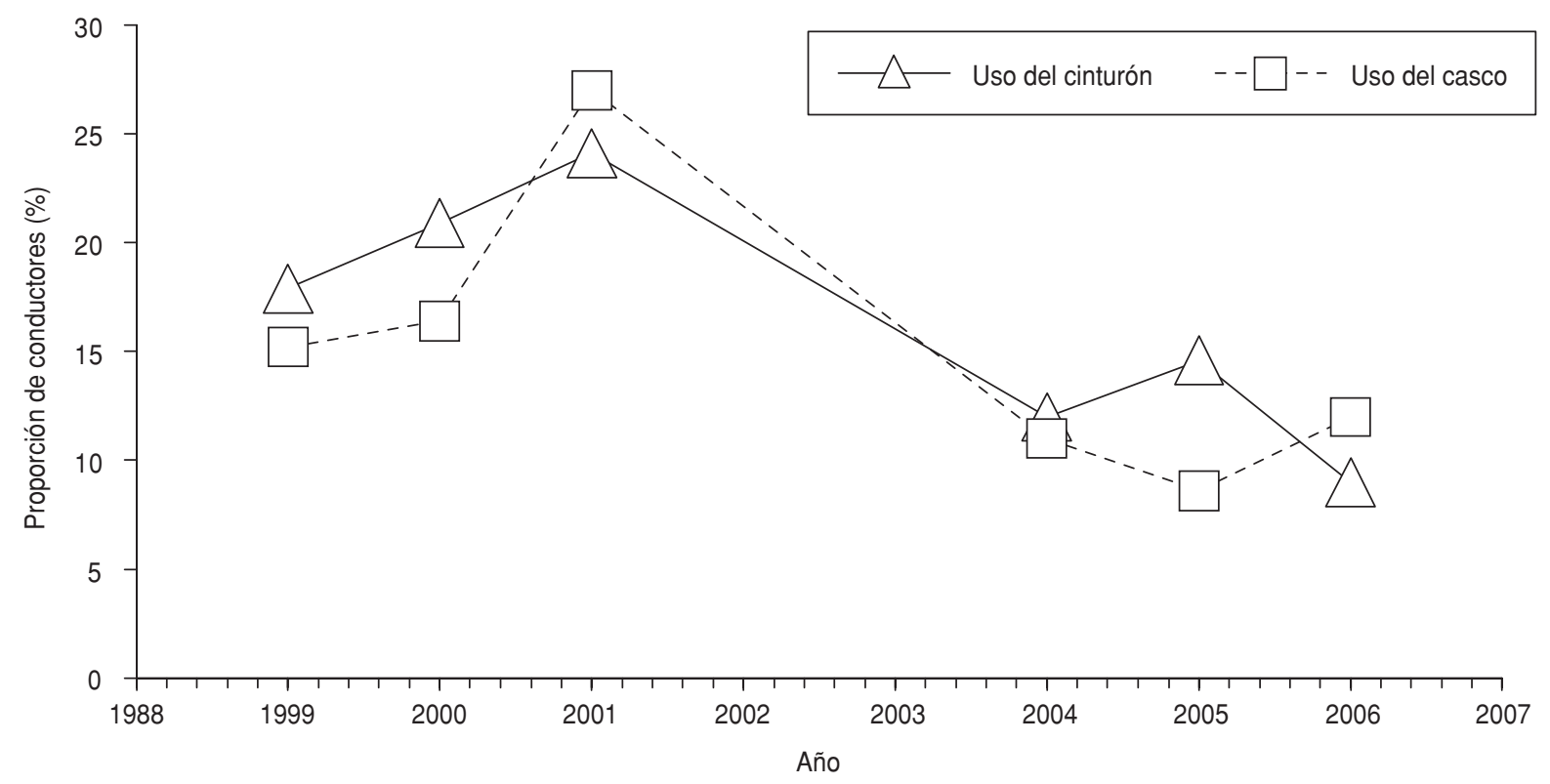

Fuente: observaciones de los autores. Total de vehículos contabilizados (automóviles/motocicletas): 1999: 3 403/548; 2000: 4 765/781; 2001: 4 777/778; 2004: 3 880/870; 2005: 3 622/1 268; 2006: 4 170/1013.

En caso de colisión, los motociclistas que no lo usan tienen una probabilidad tres veces mayor de sufrir traumatismos craneales (16).

En cuanto a la proporción relativamente importante de motocicletas que transportaban niños en este estudio $(7 \%)$, cabe mencionar que aunque esta práctica es sin duda peligrosa, muchas veces resulta la única opción para familias que viven en barrios que no cuentan con transportes públicos. Facilitar el acceso al servicio de transporte público podría evitar que pequeñas motocicletas con niños circularan al lado de camiones.

Durante las siete horas de observación, se registró el paso de 14 carros tirados por caballos. Estos vehículos sólo podrán ser erradicados cuando se resuelva el problema social de la gente que los utiliza para recoger basura; mientras tanto, los carros deberían llevar elementos reflectantes.

Se compararon los resultados obtenidos con los registrados en años anteriores por el mismo equipo observador. Se pudo así comprobar que el cumplimiento de las normas de tránsito seleccionadas para realizar este trabajo, tales como el uso de cinturones de seguridad por parte de los automovilistas y de cascos por parte de los motociclistas, se fue degradando con el paso de los años. Si bien hubo una tendencia creciente a respetarlas entre 1999 y 2001, hasta alcanzar un valor máximo de $27 \%$ en la proporción de uso del casco y del $24,1 \%$ en la de empleo del cinturón de seguridad, luego ambas cifras cayeron abruptamente de manera muy significativa $(P<0,000001)$ (figura 1). Se puede concluir que la mayoría de quienes conducen vehículos en la ciudad de Santa Fe lo hacen violando estas normas de tránsito y que los porcentajes de acatamiento de dichas normas en los tres últimos estudios son peores que los de los tres primeros.

La gran mayoría de los conductores son titulares de un permiso obtenido luego de aprobar exámenes en los que se demuestra conocer las normas de tránsito. Por lo tanto, no es el desconocimiento el motivo del incumplimiento de dichas normas por parte de la sociedad.

En los últimos siete años, las campañas de prevención de accidentes reali- zadas no han logrado fomentar un mayor acatamiento de las disposiciones de la Ley de Tránsito. No hay pruebas de que, por sí solas, la educación vial y las campañas de información sean eficaces para reducir los índices de colisiones en la vía pública. La publicidad empleada en apoyo de medidas de aplicación de la ley incrementa la eficacia de éstas, pero por sí sola influye muy poco en el comportamiento de los conductores y peatones $(17,18)$.

En Finlandia, la experiencia de treinta años de obligatoriedad del uso del cinturón de seguridad permitió comprobar que si la legislación que establece dicha obligación no va acompañada de controles y sanciones, su efecto en el porcentaje de uso del cinturón es reducido (19).

El gobierno santafesino se ha desentendido de su obligación de hacer cumplir la Ley de Tránsito y ni siquiera sus agentes la respetan cuando conducen vehículos oficiales.

Eduardo Mondino, Defensor del Pueblo de la Nación, ha declarado que la Argentina sufre una endemia de siniestros viales, a los que define como una verdadera enfermedad social (20). 
Para enfrentar esta "enfermedad endémica" es preciso aplicar una metodología equivalente a la empleada por las autoridades sanitarias para combatir las enfermedades infecciosas: además del "registro de los casos" (número de lesionados por accidentes), es necesario emplear las "vacunas" de eficacia reconocida (normas de tránsito), aplicando "inyectables" (sanciones) y controlando de manera regular los "carnés de vacunación" (evaluación periódica del acatamiento de las normas).

Para concretar esta estrategia es necesario seguir las recomendaciones que figuran en el informe elaborado conjuntament por la Organización Mundial de la Salud (OMS) y el Banco Mundial, y convertir la seguridad vial en una prioridad política, designar un organismo rector encargado de la seguridad vial, dotarlo de recursos suficientes y estipular que rinda cuentas a la sociedad (21).

\section{REFERENCIAS}

1. Asociación civil Luchemos por la Vida. Accidentes de tránsito. Estadísticas: muertos en Argentina durante 2005. Hallado en: http:// www.luchemos.org.ar/espa/mapa0005.htm. Acceso el 10 de febrero de 2006.

2. Waisman I. Los pediatras argentinos y las lesiones no intencionales en la infancia. Arch Arg Ped. 2002;100(4):275-7.

3. Barco G. La Argentina con más muertes al volante. Hallado en: http:/ / www. lanacion.com. ar/Archivo/nota.asp?nota_id=709078. Acceso el 10 de febrero de 2006.

4. Evans L. Restraint effectiveness, occupant ejection from cars and fatality reductions. Accid Anal Prev. 1990;22:167-75.

5. Silveira AJ. Seat belt use in Argentina: a 10 year struggle. Traffic Inj Prev. 2003; 4:173-5.

6. Williams AF, Wells JK, Farmer CM. Effectiveness of Ford's belt reminder system in increasing seat belt use. Inj Prev. 2002;8:293-6.

7. Williams AF, Wells JK. Driver's assessment of Ford's belt reminder system. Traffic Inj Prev. 2003;4:358-62.

8. Argentina. Ley 24.449: Ley de Tránsito. Buenos Aires: Boletín oficial, 10 de febrero de 1995. Hallado en: http://www.vialidad.gov. ar/legislacion_de_transito/Ley\%2024449.pdf. Acceso el 10 de febrero de 2006.

9. Estados Unidos, U.S. Department of Transportation, National Highway Traffic Safety
Administration. Traffic safety facts 2002: children. Washington, DC: NHTSA; 2003. (DOT HS-809-607).

10. Mohan D, Schneider L. An evaluation of adult clasping strength for restraining lap held infants. Hum factors. 1979;21:635-45.

11. Redelmeier DA, Tibshirani RJ. Association between cellular-telephone calls and motor vehicle collisions. N Engl J Med. 1997;336(7):453-8.

12. McEvoy S, Stevenson M, McCartt A, Woodward M, Haworth C, Palamara P, et al. Role of mobile phones in motor vehicle crashes resulting in hospital attendance: a case-crossover study. BMJ. 2005;331:428.

13. Asociación civil Luchemos por la vida. Los celulares en el automóvil. Revista Luchemos por la vida N. ${ }^{\circ}$ 9, 1998. Hallado en: http: / / www. luchemos.org.ar/espa/rev9/rev9p04.htm. Acceso el 10 de febrero de 2006.

14. Asociación civil Luchemos por la vida. En moto usar casco = tener cerebro. Revista Luchemos por la vida $\mathrm{N}^{\circ}$ 20, 2002. Hallado en: http:/ / www.luchemos.org.ar/espa/rev20/ pag07.htm. Acceso el 10 de febrero de 2006.

15. Servadei F, Begliomini C, Gardini E, Giustini M, Taggi F, Kraus J. Effect of Italy's motorcycle helmet law on traumatic brain injuries. Inj Prev. 2003;9:257-60.

16. Kulanthayan S, Umar RS, Hariza HA, Nasir MT, Harwant S. Compliance of proper safety helmet usage in motorcyclists. Med J Malasya. 2000;55:40-4.

17. Duperrex O, Bunn F, Roberts I. Safety education of pedestrians for injury prevention: a systematic review of randomised controlled trials. BMJ. 2002;324: 1129.

18. Ker K, Roberts I, Collier T, Renton F, Bunn F. Pos-licence driver education for the prevention of road traffic crashes. Cochrane Database Syst Rev. 2003;(3): CD003734

19. European Transport Safety Council. Seat belts and child restraints: increasing use and optimising performance. Bruselas: ETSC; 1996.

20. Argentina sufre una endemia de siniestros viales [reportaje]. El diario del mundo hospitalario (Asociación de médicos municipales de la ciudad de Buenos Aires) N. ${ }^{\circ}$ 124, 2006. Hallado en: http://www.medicos-municipales.org.ar/ repor0306.htm. Acceso el 8 de julio de 2006.

21. Organización Panamericana de la Salud. Informe mundial sobre prevención de los traumatismos causados por el tránsito. Washington, D.C.: OPS; 2004. (Publicación científica y técnica N. ${ }^{\circ} 599$ ).

Manuscrito recibido el 15 de noviembre de 2006. Aceptado para publicación el 27 de febrero de 2007.

ABSTRACT An observational, descriptive study was conducted to evaluate compliance with certain traffic regulations in city of Santa Fe, Argentina, and compare these with measurements taken in prior years. During January 2-4, 2006, at 13 sites across the city, compliance with the following traffic rules was measured: for car drivers: seat belt use and restricting minors to the rear seat; and for motorcycle drivers: helmet use and not carrying a minor on board. At total of 4173 cars and 1013 motorcycles were observed. Only $9 \%$ of the car drivers wore seatbelts. Of the 246 cars carrying minors, $56 \%$ had a minor in the front seat. Regarding the motorcycles drivers, only $12 \%$ wore a helmet and $6.7 \%$ had a child on board. When these observations were compared with those of the previous five years, findings showed that over the past three years compliance rates had decreased. The results of this study suggest that most drivers in city of Santa Fe do not obey the stated traffic laws. In order to reduce the high rate of mortality from traffic accidents, in addition to legislation and public-awareness campaigns, a system for enforcing compliance is needed.

Key words Accidents, traffic; accident prevention; seat belts; Argentina. 